\title{
EMBEDDINGS BETWEEN WEIGHTED TANDORI AND CESÀRO FUNCTION SPACES
}

\author{
Tuğçe ÜNVER YILDIZ \\ Department of Mathematics, Faculty of Science and Arts, Kirikkale University, Kirikkale, \\ TURKEY
}

ABSTRACT. We characterize the weights for which the two-operator inequality

$$
\left\|\left(\int_{0}^{x} f(t)^{p} v(t)^{p} d t\right)^{\frac{1}{p}}\right\|_{q, u,(0, \infty)} \leq c\left\|\operatorname{esssup}_{t \in(x, \infty)} f(t)\right\|_{r, w,(0, \infty)}
$$

holds for all non-negative measurable functions on $(0, \infty)$, where $0<p<q \leq$ $\infty$ and $0<r<\infty$, namely, we find the least constants in the embeddings between weighted Tandori and Cesàro function spaces. We use the combination of duality arguments for weighted Lebesgue spaces and weighted Tandori spaces with weighted estimates for the iterated integral operators.

\section{INTRODUCTION}

Given two function spaces $X, Y$ and an operator $\mathrm{T}$, a standard problem is characterizing the conditions for which T maps $X$ into $Y$. If $X$ and $Y$ are (quasi) Banach spaces of measurable functions, a bounded operator $\mathrm{T}: X \rightarrow Y$ satisfies the inequality $\|\mathrm{T} f\|_{Y} \leq c\|f\|_{X}$ for all $f \in X$ where $c \in(0, \infty)$. When $T$ is the identity operator I, we say that $X$ is embedded into $Y$ and write $X \hookrightarrow Y$. The least constant $c$ in the embedding $X \hookrightarrow Y$ is $\|\mathrm{I}\|_{X \rightarrow Y}$.

In this paper, we find the optimal constants in the embedding between weighted Tandori and Cesàro function spaces. We shall begin with the definitions of the function spaces considered in this paper.

Given a measurable function $f$ on $E$, we set

$$
\|f\|_{p, E}:=\left(\int_{E}|f(x)|^{p} d x\right), \quad 1 \leq p<\infty
$$

2020 Mathematics Subject Classification. Primary 46E30; Secondary 26D10.

Keywords and phrases. Cesàro function spaces, Copson function spaces, Tandori function spaces, embeddings, weighted inequalities, Hardy operator, Copson operator, iterated operators.

tugceunver@kku.edu.tr

(D) 0000-0003-0414-8400.

(C)2021 Ankara University Communications Faculty of Sciences University of Ankara-Series A1 Mathematics and Statistics 
and

$$
\|f\|_{\infty, E}:=\operatorname{essup}_{x \in E}|f(x)|, \quad p=\infty .
$$

If $w$ is a weight on $E$, that is, measurable, positive and finite a.e. on $E$, then we denote by $L_{p, w}(E)$, the weighted Lebesgue space, the set of measurable functions satisfying $\|f\|_{p, w, E}:=\|f w\|_{p, E}<\infty$.

Let $0<p, q \leq \infty, u$ be a non-negative measurable function and $v$ be a weight, the weighted Cesàro space $\operatorname{Ces}_{p, q}(u, v)$ is the set of all measurable functions such that $\|f\|_{\operatorname{Ces}_{p, q}(u, v)}<\infty$, where

$$
\|f\|_{\operatorname{Ces}_{p, q}(u, v)}:=\|\| f\left\|_{p, v,(0, x)}\right\|_{q, u,(0, \infty)}
$$

and the weighted Copson space $\operatorname{Cop}_{p, q}(u, v)$ is the set of all measurable functions such that $\|f\|_{\operatorname{Cop}_{p, q}(u, v)}<\infty$, where

$$
\|f\|_{\operatorname{Cop}_{p, q}(u, v)}:=\|\| f\left\|_{p, v,(x, \infty)}\right\|_{q, u,(0, \infty)} .
$$

The classical Cesàro spaces $\operatorname{Ces}_{1, p}\left(x^{-1}, 1\right), 1 \leq p<\infty$ were defined by Shiue 20 in 1970. When $1<p<\infty$ Hassard and Hussein 12 proved that $\operatorname{Ces}_{1, p}\left(x^{-1}, 1\right)$ are separable Banach spaces and Bennett $\left[4\right.$ showed that the spaces $\operatorname{Ces}_{1, p}\left(x^{-1}, 1\right)$ and $\operatorname{Cop}_{1, p}\left(1, x^{-1}\right)$ coincide. Dual spaces of the classical Cesàro function spaces were considered in 4, 21. In 1], factorization theorems for classical Cesàro function spaces were given and based on these results the dual spaces of classical Cesàro function spaces were presented. One weighted Cesàro function spaces $\operatorname{Ces}_{1, p}\left(w^{\frac{1}{p}}, 1\right)$ and their duals were considered in [13. Recently, in 3], factorization of the spaces $\operatorname{Ces}_{1, p}\left(x^{-1} w^{\frac{1}{p}}, 1\right)$ and $\operatorname{Cop}_{1, p}\left(w^{\frac{1}{p}}, x^{-1}\right)$ are given.

We do not aim to give a thorough set of references on the history of these spaces. Instead, we refer the interested reader to survey paper [2], where the comprehensive history on the structure of Cesàro and Copson function spaces are given.

In this paper our primary focus is the following inequality

$$
\|f\|_{\operatorname{Ces}_{p_{2}, q_{2}}\left(u_{2}, v_{2}\right)} \leq c\|f\|_{\operatorname{Cop}_{p_{1}, q_{1}}\left(u_{1}, v_{1}\right)}
$$

for all measurable functions where $0<p_{i}, q_{i} \leq \infty, i=1,2$.

There is more than one motivation to study inclusion between Cesàro and Copson spaces. First of all when $p_{1}=q_{1}$ or $p_{2}=q_{2}$, weighted Cesàro and Copson function spaces coincide with some weighted Lebesgue spaces (see 9 , Lemmas 3.4$3.5]$ ), thus inequality (1) is a generalization of the well-known weighted direct and reverse Hardy-type inequalities (e.g. [15, 7, 19]). Another justification is to give the characterization of pointwise multipliers between two spaces of Cesàro and Copson type, because it reduces to the characterization the embeddings between these spaces. In [11, Section 7] Grosse-Erdmann considered the multipliers between the spaces of $p$-summable sequences and Cesàro and Copson sequence spaces. He also introduced corresponding function spaces but the characterization of the multipliers

\footnotetext{
${ }^{1}$ When $E=(0, \infty)$, we simply write $L_{p, w}$ instead of $L_{p, w}(0, \infty)$.
} 
between two spaces of Cesàro and Copson type remained open for both sequence and function spaces for a long time.

The characterization of the inequality $(1)$ is given in one parameter case when $p_{1}=p_{2}=1, q_{1}=q_{2}=p>1, v_{1}(t)=t^{-\beta-1}, v_{2}(t)=t^{\alpha-1}, u_{1}(t)=t^{\beta-1 / p}$ and $u_{2}(t)=t^{-\alpha-1 / p}, t>0, \alpha, \beta>0$ in 5 . Moreover, it was shown that the inequality is reversed when $0<p<1$. In 6 , inequality (1) is considered for two different parameters in the special case $p_{1}=p_{2}=1, q_{1}=p, q_{2}=q, v_{1}(t)=t^{-1}, v_{2}(t)=1$, $u_{1}(t)^{p}=v(t), u_{2}(t)^{q}=w(t) t^{-q}, t>0$, under the restriction $q \geq 1$ in order to characterize the embeddings between some Lorentz-type spaces. Recently, in 9 the two sided estimates for the best constant in (1) is given for four weights and four parameters $0<p_{1}, p_{2}, q_{1}, q_{2}<\infty$ under the restriction $p_{2} \leq q_{2}$. Moreover, using these results, in [9. Theorems 3.11-3.12], the associate spaces of weighted Copson and Cesàro function spaces were characterized and in 10] pointwise multipliers between Cesàro and Copson function spaces are given for some ranges of parameters.

Furthermore, in 2015, Lesnik and Maligranda 16 17 began studying these spaces within an abstract framework, where they used a more general function space $X$ instead of the weighted Lebesgue spaces. When $X$ is a Banach space, they defined Cesàro space $C X$, Copson space $C^{*} X$ and Tandori space $\widetilde{X}$ as the set of all measurable functions, respectively, with the following norms:

$$
\begin{aligned}
\|f\|_{C X} & =\left\|\frac{1}{x} \int_{0}^{x}|f(t)| d t\right\|_{X}<\infty, \\
\|f\|_{C^{*} X} & =\left\|\int_{x}^{\infty} \frac{|f(t)|}{t} d t\right\|_{X}<\infty, \\
\|f\|_{\tilde{X}} & =\left\|\operatorname{ess~sup~}_{t \in(x, \infty)}|f(t)|\right\|_{X}<\infty .
\end{aligned}
$$

In 18, they named $\tilde{X}$ as the generalized Tandori spaces in honour of Tandori who provided dual spaces to the spaces $C L_{\infty}[0,1]$ in 22$]$. Their definition is related to our definition in the following way:

$$
C L_{p, w}=\operatorname{Ces}_{1, p}\left(x^{-1} w(x), 1\right), \quad C^{*} L_{p, w}=\operatorname{Cop}_{1, p}\left(w, x^{-1}\right), \quad \widetilde{L}_{p, w}=\operatorname{Cop}_{\infty, p}(w, 1) .
$$

We should note that recently in 14 multipliers between $C L_{p}$ and $C L_{q}$ are given when $1<q \leq p \leq \infty$.

We want to continue this research. In this paper, we will handle the inequality (1) when $p_{1}=\infty$. In other words, we will consider the embeddings $\widetilde{L}_{r, w} \hookrightarrow \operatorname{Ces}_{p, q}(u, v)$, namely, we will give the characterization of the following inequality,

$$
\|f\|_{\operatorname{Ces}_{p, q}(u, v)} \leq C\|f\|_{\widetilde{L}_{r, w}}
$$

for all measurable functions where $p, q, r \in(0, \infty)$ with $p<q$. The restriction on the parameters arises from the duality argument. The key ingredient of the proof is combining characterizations of the associate spaces of Tandori spaces, namely, the 
reverse Hardy-type inequality for supremal operators which was given in 19 with the characterizations of some iterated Hardy-type inequalities.

Throughout the paper, we put $0 \cdot \infty=\frac{0}{0}=0$. We write $A \approx B$ if there exist positive constants $\alpha, \beta$ independent of relevant quantities appearing in expressions $\mathrm{A}$ and $\mathrm{B}$ such that

holds.

$$
\alpha \leq \frac{A}{B} \leq \beta
$$

The symbol $\mathfrak{M}$ will stand for the set of all measurable functions on $(0, \infty)$, and we denote the class of non-negative elements of $\mathfrak{M}$ by $\mathfrak{M}^{+}$.

We sometimes omit the differential element $d x$ to make the formulas simpler when the expressions are too long.

The paper is structured as follows. In Section 2, we formulate the main results of this paper. In Section 3, we collect some properties and necessary background material. Finally, in the last section, we give the proofs of our main results.

\section{MAIN RESULTS}

It is convenient to start this section by recalling some properties of the weighted Cesàro and Copson spaces. Let $0<p, q \leq \infty$. Assume that $u$ is a non-negative measurable function and $v$ is a weight. We will always assume that $\|u\|_{q,(t, \infty)}<\infty$ for all $t>0$ and $\|u\|_{q,(0, t)}<\infty$ for all $t>0$, when considering weighted Cesàro and Copson function spaces, respectively. Otherwise, these spaces consist only of functions equivalent to zero (see, 9 , Lemmas 3.1-3.2]).

In this section, we will formulate the least constant in the embedding

$$
\widetilde{L}_{r, w} \hookrightarrow \operatorname{Ces}_{p, q}(u, v) .
$$

Remark 1. Observe that,

$$
\|\mathrm{I}\|_{\operatorname{Cop}_{\infty, r}\left(w, v_{1}\right) \rightarrow \operatorname{Ces}_{p, q}\left(u, v_{2}\right)}=\|\mathrm{I}\|_{\widetilde{L}_{r, w} \rightarrow \operatorname{Ces}_{p, q}\left(u, \frac{v_{2}}{v_{1}}\right)}
$$

holds. Therefore, it is enough to consider the three weighted case (3).

Remark 2. Note that, when $p=q$ or $r=\infty$, this problem is not interesting since it reduces to the characterizations of Hardy-type inequalities and can be found in [9], therefore we will consider the cases when $r<\infty$. On the other hand, we have the restriction $p<q$, which arises from the duality argument.

Now we are in position to formulate the results of this paper. We begin with the cases where $q=\infty$.

Theorem 3. Let $0<p, r<\infty$. Assume that $v$ is a weight, $w \in \mathfrak{M}^{+}$such that $\|w\|_{r,(0, t)}<\infty$ for all $t \in(0, \infty)$ and $w \neq 0$ a.e. on $(0, \infty)$, and $u \in \mathfrak{M}^{+}$such that $\|u\|_{\infty,(t, \infty)}<\infty$ for all $t \in(0, \infty)$.

(i) If $r \leq p$, then

$$
\|\mathrm{I}\|_{\widetilde{L}_{r, w} \rightarrow \operatorname{Ces}_{p, \infty}(u, v)} \approx I_{1},
$$


where

$$
I_{1}:=\operatorname{ess}_{x \in(0, \infty)} u(x) \sup _{t \in(0, x)}\left(\int_{0}^{t} v^{p}\right)^{\frac{1}{p}}\left(\int_{0}^{t} w^{r}\right)^{-\frac{1}{r}}<\infty .
$$

(ii) If $p<r$, then

$$
\|\mathrm{I}\|_{\widetilde{L}_{r, w} \rightarrow \operatorname{Ces}_{p, \infty}(u, v)} \approx I_{2}+I_{3}+I_{4}
$$

where

$$
\begin{aligned}
& I_{2}:=\operatorname{esssup}_{x \in(0, \infty)} u(x)\left(\int_{0}^{x}\left(\int_{0}^{t} v^{p}\right)^{\frac{r}{r-p}}\left(\int_{0}^{t} w^{r}\right)^{-\frac{r}{r-p}} w(t)^{r} d t\right)^{\frac{r-p}{r p}}<\infty, \\
& I_{3}:=\operatorname{ess~sup}_{x \in(0, \infty)} u(x)\left(\int_{x}^{\infty}\left(\int_{0}^{t} w^{r}\right)^{-\frac{r}{r-p}} w(t)^{r} d t\right)^{\frac{r-p}{r p}}\left(\int_{0}^{x} v^{p}\right)^{\frac{1}{p}}<\infty,
\end{aligned}
$$

and

$$
I_{4}:=\left(\int_{0}^{\infty} w^{r}\right)^{-\frac{1}{r}} \operatorname{esssup}_{x \in(0, \infty)} u(x)\left(\int_{0}^{x} v^{p}\right)^{\frac{1}{p}}<\infty .
$$

When $q<\infty$, we consider the cases $r \leq p$ and $p<r$ separately.

Theorem 4. Let $0<r \leq p<q<\infty$. Assume that $v \in \mathfrak{M}^{+}, w \in \mathfrak{M}^{+}$such that $\|w\|_{r,(0, t)}<\infty$ for all $t \in(0, \infty)$ and $w \neq 0$ a.e. on $(0, \infty)$, and $u \in \mathfrak{M}^{+}$such that $\|u\|_{q,(t, \infty)}<\infty$ for all $t \in(0, \infty)$. Then

$$
\|\mathrm{I}\|_{\widetilde{L}_{r, w} \rightarrow \operatorname{Ces}_{p, q}(u, v)} \approx I_{5}+I_{6},
$$

where

$$
I_{5}:=\sup _{t \in(0, \infty)}\left(\int_{0}^{t} w(s)^{r} d s\right)^{-\frac{1}{r}}\left(\int_{0}^{t}\left(\int_{0}^{s} v(y)^{p} d y\right)^{\frac{q}{p}} u(s)^{q} d s\right)^{\frac{1}{q}}<\infty,
$$

and

$$
I_{6}:=\sup _{t \in(0, \infty)}\left(\int_{0}^{t} w(s)^{r} d s\right)^{-\frac{1}{r}}\left(\int_{0}^{t} v(s)^{p} d s\right)^{\frac{1}{p}}\left(\int_{t}^{\infty} u(s)^{q} d s\right)^{\frac{1}{q}}<\infty .
$$

Theorem 5. Let $0<p<r<\infty$ and $0<p<q<\infty$. Assume that $v \in \mathfrak{M}^{+}$, such that $v>0,\|v\|_{p,(0, t)}<\infty$ for all $t \in(0, \infty)$ and $\|v\|_{p,(0, \infty)}=\infty$. Suppose that $w \in \mathfrak{M}^{+}$such that $\|w\|_{r,(0, t)}<\infty$ for all $t \in(0, \infty)$ and $w \neq 0$ a.e. on $(0, \infty)$, and $u \in \mathfrak{M}^{+}$such that $\|u\|_{q,(t, \infty)}<\infty$ for all $t \in(0, \infty)$. Let

- $\int_{0}^{t}\left(\int_{0}^{s} v^{p}\right)^{\frac{r}{r-p}}\left(\int_{0}^{s} w^{r}\right)^{-\frac{r}{r-p}} w(s)^{r} d s<\infty$ for all $t \in(0, \infty)$,

- $\int_{0}^{1}\left(\int_{0}^{s} w^{r}\right)^{-\frac{r}{r-p}} w(s)^{r} d s=\infty$, 
- $\int_{t}^{\infty}\left(\int_{0}^{s} w^{r}\right)^{-\frac{r}{r-p}} w(s)^{r} d s<\infty$ for all $t \in(0, \infty)$,

- $\int_{1}^{\infty}\left(\int_{0}^{s} v^{p}\right)^{\frac{r}{r-p}}\left(\int_{0}^{s} w^{r}\right)^{-\frac{r}{r-p}} w(s)^{r} d s=\infty$

hold.

(i) If $r \leq q$, then

$$
\|\mathrm{I}\|_{\widetilde{L}_{r, w} \rightarrow \operatorname{Ces}_{p, q}(u, v)} \approx I_{7}+I_{8}+I_{9},
$$

where

$$
\begin{gathered}
I_{7}:=\left(\int_{0}^{\infty} w^{r}\right)^{-\frac{1}{r}}\left(\int_{0}^{\infty}\left(\int_{0}^{y} v(s)^{p} d s\right)^{\frac{q}{p}} u(y)^{q} d y\right)^{\frac{1}{q}}<\infty \\
I_{8}:=\sup _{x \in(0, \infty)}\left(\int_{0}^{x}\left(\int_{0}^{t} v^{p}\right)^{\frac{r}{r-p}}\left(\int_{0}^{t} w^{r}\right)^{-\frac{r}{r-p}} w(t)^{r} d t\right)^{\frac{r-p}{r p}}\left(\int_{x}^{\infty} u^{q}\right)^{\frac{1}{q}}<\infty,
\end{gathered}
$$

and

$$
I_{9}:=\sup _{x \in(0, \infty)}\left(\int_{x}^{\infty}\left(\int_{0}^{t} w^{r}\right)^{-\frac{r}{r-p}} w(t)^{r} d t\right)^{\frac{r-p}{r p}}\left(\int_{0}^{x}\left(\int_{0}^{t} v^{p}\right)^{\frac{q}{p}} u(t)^{q} d t\right)^{\frac{1}{q}}<\infty .
$$

(ii) If $q<r$, then

$$
\|\mathrm{I}\|_{\tilde{L}_{r, w} \rightarrow \operatorname{Ces}_{p, q}(u, v)} \approx I_{7}+I_{10}+I_{11},
$$

where $I_{7}$ is defined in (4),

$$
\begin{aligned}
I_{10}:=\left(\int_{0}^{\infty}\left(\int_{x}^{\infty} u^{q}\right)^{\frac{r}{r-q}}\left(\int_{0}^{x}\left(\int_{0}^{t} v^{p}\right)^{\frac{r}{r-p}}\left(\int_{0}^{t} w^{r}\right)^{-\frac{r}{r-p}} w(t)^{r} d t\right)^{\frac{r(q-p)}{p(r-q)}}\right. \\
\left.\times\left(\int_{0}^{x} v^{p}\right)^{\frac{r}{r-p}}\left(\int_{0}^{x} w^{r}\right)^{-\frac{r}{r-p}} w(x)^{r} d x\right)^{\frac{r-q}{r q}}<\infty,
\end{aligned}
$$

and

$$
\begin{aligned}
I_{11}:=\left(\int_{0}^{\infty}\left(\int_{0}^{x}\left(\int_{0}^{t} v^{p}\right)^{\frac{q}{p}} u(t)^{q} d t\right)^{\frac{r}{r-q}}\left(\int_{x}^{\infty}\left(\int_{0}^{t} w^{r}\right)^{-\frac{r}{r-p}} w(t)^{r} d t\right)^{\frac{r(q-p)}{p(r-q)}}\right. \\
\left.\times\left(\int_{0}^{x} w^{r}\right)^{-\frac{r}{r-p}} w(x)^{r} d x\right)^{\frac{r-q}{r q}}<\infty .
\end{aligned}
$$

\section{BACKGROUND MATERIAL}

In this section we quote some known results. Let us start with the characterization of the reverse Hardy-type inequality for supremal operator, that is,

$$
\left(\int_{0}^{\infty} f(t)^{p} \mathfrak{u}(t)^{p} d t\right)^{\frac{1}{p}} \leq C\left(\int_{0}^{\infty} \mathfrak{w}(t)^{q}\left(\operatorname{esssup}_{s \in(t, \infty)} f(s)\right)^{q} d t\right)^{\frac{1}{q}}
$$


for all non-negative measurable functions $f$ on $(0, \infty)$ where $0<p, q<\infty$.

Theorem 6. [19, Theorem 3.4] Let $0<p, q<\infty$. Assume that $\mathfrak{u} \in \mathfrak{M}^{+}$and $\mathfrak{w} \in \mathfrak{M}^{+}$such that $\int_{0}^{t} \mathfrak{w}^{q}<\infty$ for all $t \in(0, \infty)$ and $\mathfrak{w} \neq 0$ a.e. on $(0, \infty)$.

(i) If $q \leq p$, then inequality (5) holds for all non-negative measurable functions $f$ on $(0, \infty)$ if and only if $A_{1}<\infty$, where

$$
A_{1}:=\sup _{t \in(0, \infty)}\left(\int_{0}^{t} \mathfrak{u}^{p}\right)^{\frac{1}{p}}\left(\int_{0}^{t} \mathfrak{w}^{q}\right)^{-\frac{1}{q}} .
$$

Moreover, the least possible constant $C$ in (5) satifies $C \approx A_{1}$.

(ii) If $p<q$, then inequality (5) holds for all non-negative measurable functions $f$ on $(0, \infty)$ if and only if $A_{2}<\infty$ and $A_{3}<\infty$, where

$$
A_{2}:=\left(\int_{0}^{\infty}\left(\int_{0}^{t} \mathfrak{u}^{p}\right)^{\frac{q}{q-p}}\left(\int_{0}^{t} \mathfrak{w}^{q}\right)^{-\frac{q}{q-p}} \mathfrak{w}(t)^{q} d t\right)^{\frac{q-p}{p q}}
$$

and

$$
A_{3}:=\left(\int_{0}^{\infty} \mathfrak{u}^{p}\right)^{\frac{1}{p}}\left(\int_{0}^{\infty} \mathfrak{w}^{q}\right)^{-\frac{1}{q}}
$$

Moreover, the least possible constant $C$ in (5) satifies $C \approx A_{2}+A_{3}$.

We next recall the characterization of the weighted iterated inequality involving Hardy and Copson operators, that is,

$$
\left(\int_{0}^{\infty}\left(\int_{0}^{t}\left(\int_{s}^{\infty} g\right) \mathfrak{v}(s) d s\right)^{q} \mathfrak{w}(t)^{q} d t\right)^{\frac{1}{q}} \leq C\left(\int_{0}^{\infty} g(t)^{p} \mathfrak{u}(t)^{p} d t\right)^{\frac{1}{p}} .
$$

Note that the characterization of inequality (9) is given in 8 . In the next theorem, we provide a modified version of 8 , Theorem 3.1], using the gluing lemmas presented in the recent paper 10]. Denote by

$$
\mathcal{V}(t):=\int_{0}^{t} \mathfrak{v}(s) d s, t>0 .
$$

Theorem 7. Let $1<p<\infty$ and $0<q<\infty$. Assume that $\mathfrak{u} \in \mathfrak{M}^{+}$and $\mathfrak{v}, \mathfrak{w} \in \mathfrak{M}^{+}$ such that $\mathfrak{v}(t)>0, \mathcal{V}(t)<\infty$ for all $t \in(0, \infty)$ and $\mathcal{V}(\infty)=\infty$,

- $\int_{0}^{t} \mathcal{V}(s)^{q} \mathfrak{w}(s)^{q} d s<\infty$ for all $t \in(0, \infty)$ and $\int_{1}^{\infty} \mathcal{V}(s)^{q} \mathfrak{w}(s)^{q} d s=\infty$,

- $\int_{t}^{\infty} \mathfrak{w}(s)^{q} d s<\infty$ for all $t \in(0, \infty)$ and $\int_{0}^{1} \mathfrak{w}(s)^{q} d s=\infty$.

(i) If $p \leq q$, then (9) holds for all non-negative measurable functions $f$ on $(0, \infty)$ if and only if $B_{1}<\infty$ and $B_{2}<\infty$, where

$$
B_{1}:=\sup _{x \in(0, \infty)}\left(\int_{0}^{x} \mathcal{V}(t)^{q} \mathfrak{w}(t)^{q} d t\right)^{\frac{1}{q}}\left(\int_{x}^{\infty} \mathfrak{u}(t)^{-\frac{p}{p-1}} d t\right)^{\frac{p-1}{p}}
$$


and

$$
B_{2}:=\sup _{x \in(0, \infty)}\left(\int_{x}^{\infty} \mathfrak{w}(t)^{q} d t\right)^{\frac{1}{q}}\left(\int_{0}^{x} \mathcal{V}(t)^{\frac{p}{p-1}} \mathfrak{u}(t)^{-\frac{p}{p-1}} d t\right)^{\frac{p-1}{p}} .
$$

Moreover, the least possible constant $C$ in $(9)$ satifies $C \approx B_{1}+B_{2}$.

(ii) If $q<p$, then (9) holds for all non-negative measurable functions $f$ on $(0, \infty)$ if and only if $B_{3}<\infty$ and $B_{4}<\infty$, where

$$
B_{3}:=\left(\int_{0}^{\infty}\left(\int_{x}^{\infty} \mathfrak{u}(t)^{-\frac{p}{p-1}} d t\right)^{\frac{q(p-1)}{p-q}}\left(\int_{0}^{x} \mathcal{V}(t)^{q} \mathfrak{w}(t)^{q} d t\right)^{\frac{q}{p-q}} \mathcal{V}(x)^{q} \mathfrak{w}(x)^{q} d x\right)^{\frac{p-q}{p q}}
$$

and

$$
B_{4}:=\left(\int_{0}^{\infty}\left(\int_{x}^{\infty} \mathfrak{w}(t)^{q} d t\right)^{\frac{q}{p-q}}\left(\int_{0}^{x} \mathcal{V}(t)^{\frac{p}{p-1}} \mathfrak{u}(t)^{-\frac{p}{p-1}} d t\right)^{\frac{q(p-1)}{p-q}} \mathfrak{w}(x)^{q} d x\right)^{\frac{p-q}{p q}} .
$$

Moreover, the least possible constant $C$ in (9) satifies $C \approx B_{3}+B_{4}$.

Proof. The proof is the combination of [8, Theorem 3.1, (iii)] and 10, Lemma 2.7] for the first case and [8, Theorem 3.1, (iv)] and [10, Lemma 2.8] for the second case.

\section{PROOFS}

Denote by

$$
\mathcal{R}(p, r ; v, w):=\sup _{f \in \mathfrak{M}^{+}} \frac{\|f\|_{p, v,(0, \infty)}}{\left\|\operatorname{ess~sup~}_{s \in(t, \infty)} f(s)\right\|_{r, w,(0, \infty)}} .
$$

Proof of Theorem 3 Let $0<p, r<\infty$. We have

$$
C=\sup _{f \in \mathfrak{M}} \frac{\|f\|_{\operatorname{Ces}_{p, \infty}(u, v)}}{\|f\|_{\widetilde{L}_{r, w}}}=\sup _{f \in \mathfrak{M}^{+}} \frac{\operatorname{ess~sup}_{x \in(0, \infty)} u(x)\|f\|_{p, v,(0, x)}}{\left\|\operatorname{esssup}_{s \in(t, \infty)} f(s)\right\|_{r, w,(0, \infty)}} .
$$

Fix $x \in(0, \infty)$, then

$$
C=\sup _{f \in \mathfrak{M}^{+}} \frac{\operatorname{ess~sup~} u(x)\left\|f(0, \infty)_{x \in(0, x)}\right\|_{p, v,(0, \infty)}}{\left\|\operatorname{esssup}_{s \in(t, \infty)} f(s)\right\|_{r, w,(0, \infty)}} .
$$

Observe that, interchanging supremum gives

$$
C=\operatorname{esssup}_{x \in(0, \infty)} u(x) \mathcal{R}(p, r ; \tilde{v}, w),
$$

where $\tilde{v}(t)=\chi_{(0, x)}(t) v(t), t \in(0, \infty)$. Thus, the problem is reduced to the characterization of reverse Hardy-type inequalities for supremal operator. It remains to apply [Theorem 6, (i)] when $r \leq p$ and [Theorem 6, (ii)] when $p<r$. 
Proof of Theorem 4 Let $0<r \leq p<q<\infty$. We have

$$
C=\sup _{f \in \mathfrak{M}} \frac{\|f\|_{\operatorname{Ces}_{p, q}(u, v)}}{\|f\|_{\widetilde{L}_{r, w}}}
$$

Since $q / p \in(1, \infty)$, by the duality in weighted Lebesgue spaces, we have

$$
\|f\|_{\operatorname{Ces}_{p, q}(u, v)}^{p}=\sup _{g \in \mathfrak{M}^{+}} \frac{\int_{0}^{\infty}\left(\int_{0}^{t} f(s)^{p} v(s)^{p} d s\right) g(t) d t}{\left(\int_{0}^{\infty} g(t)^{\frac{q}{q-p}} u(t)^{-\frac{q p}{q-p}} d t\right)^{\frac{q-p}{q}}} .
$$

Interchanging supremum and Fubini's Theorem gives that

$$
\begin{aligned}
& C=\sup _{g \in \mathfrak{M}^{+}} \frac{1}{\left(\int_{0}^{\infty} g(t)^{\frac{q}{q-p}} u(t)^{-\frac{q p}{q-p}} d t\right)^{\frac{q-p}{q p}}} \sup _{f \in \mathfrak{M}^{+}} \frac{\left(\int_{0}^{\infty} f(s)^{p} v(s)^{p} \int_{s}^{\infty} g(t) d t d s\right)^{\frac{1}{p}}}{\left(\int _ { 0 } ^ { \infty } \left(\begin{array}{l}
\operatorname{ess} \sup f(t, \infty) \\
\left.s \in(s))^{r} w(t)^{r} d t\right)^{\frac{1}{r}}
\end{array}\right.\right.} \\
& =: \sup _{g \in \mathfrak{M}^{+}} \frac{\mathcal{R}(p, r ; \tilde{v}, w)}{\|g\|^{\frac{1}{p}}}
\end{aligned}
$$

where, $\tilde{v}(s)=v(s)\left(\int_{s}^{\infty} g(t) d t\right)^{\frac{1}{p}}, s \in(0, \infty)$, and

$$
\|g\|:=\left(\int_{0}^{\infty} g(t)^{\frac{q}{q-p}} u(t)^{-\frac{q p}{q-p}} d t\right)^{\frac{q-p}{q}} .
$$

Note that $\mathcal{R}(p, r ; \tilde{v}, w)$ is the best constant in the inequality

$$
\left(\int_{0}^{\infty} h(s)^{p} v(s)^{p} \int_{s}^{\infty} g(t) d t d s\right)^{\frac{1}{p}} \leq c\left(\int_{0}^{\infty}(\underset{s \in(t, \infty)}{\operatorname{esssup}} h(s))^{r} w(t)^{r} d t\right)^{\frac{1}{r}}, h \in \mathfrak{M}^{+}
$$

for every fixed $g \in \mathfrak{M}^{+}$. Now, we can apply Theorem 6 by taking the parameters $p, r$, and weights

$$
\mathfrak{w}(s)=w(s) \quad \mathfrak{u}(s)=v(s)\left(\int_{s}^{\infty} g\right)^{\frac{1}{p}}, \quad s>0 .
$$

Since $r \leq p$, according to the first case in Theorem 6 .

$$
\mathcal{R}(p, r ; \tilde{v}, w) \approx \sup _{t \in(0, \infty)}\left(\int_{0}^{t} v(s)^{p}\left(\int_{s}^{\infty} g\right) d s\right)^{\frac{1}{p}}\left(\int_{0}^{t} w(s)^{r} d s\right)^{-\frac{1}{r}}
$$


holds. Thus,

$$
C \approx \sup _{g \in \mathfrak{M}^{+}} \frac{\sup _{t \in(0, \infty)}\left(\int_{0}^{t} v(s)^{p}\left(\int_{s}^{\infty} g\right) d s\right)^{\frac{1}{p}}\left(\int_{0}^{t} w(s)^{r} d s\right)^{-\frac{1}{r}}}{\|g\|^{\frac{1}{p}}} .
$$

Interchanging suprema yields that

$$
C \approx \sup _{t \in(0, \infty)}\left(\int_{0}^{t} w(s)^{r} d s\right)^{-\frac{1}{r}} \sup _{g \in \mathfrak{M}^{+}} \frac{\left(\int_{0}^{\infty} v(s)^{p}\left(\int_{s}^{\infty} g\right) \chi_{(0, t)}(s) d s\right)^{\frac{1}{p}}}{\|g\|^{\frac{1}{p}}} .
$$

From Fubini's Theorem and duality in weighted Lebesgue spaces with $q / p \in(1, \infty)$ again, it follows that

$$
\begin{aligned}
C & =\sup _{t \in(0, \infty)}\left(\int_{0}^{t} w(s)^{r} d s\right)^{-\frac{1}{r}} \sup _{g \in \mathfrak{M}^{+}} \frac{\left(\int_{0}^{\infty} g(y)\left(\int_{0}^{y} v(s)^{p} \chi_{(0, t)}(s) d s\right) d y\right)^{\frac{1}{p}}}{\left(\int_{0}^{\infty} g(y)^{\frac{q}{q-p}} u(y)^{-\frac{q p}{q-p}} d y\right)^{\frac{q-p}{q p}}} \\
& \approx \sup _{t \in(0, \infty)}\left(\int_{0}^{t} w(s)^{r} d s\right)^{-\frac{1}{r}}\left(\int_{0}^{\infty}\left(\int_{0}^{y} v(s)^{p} \chi_{(0, t)}(s) d s\right)^{\frac{q}{p}} u(y)^{q} d y\right)^{\frac{1}{q}}
\end{aligned}
$$

Observe that,

$$
\begin{aligned}
\int_{0}^{\infty} & \left(\int_{0}^{y} v(s)^{p} \chi_{(0, t)}(s) d s\right)^{\frac{q}{p}} u(y)^{q} d y \\
& =\int_{0}^{t}\left(\int_{0}^{y} v(s)^{p} d s\right)^{\frac{q}{p}} u(y)^{q} d y+\left(\int_{0}^{t} v(s)^{p} d s\right)^{\frac{q}{p}}\left(\int_{t}^{\infty} u(y)^{q} d y\right) .
\end{aligned}
$$

Thus we arrive at $C \approx I_{5}+I_{6}$.

Proof of Theorem 5 Let $0<p<r<\infty$ and $0<p<q<\infty$. Using the steps identical to the preceding proof, which relies on $q / p \in(1, \infty)$, duality in weighted Lebesgue spaces, and Fubini's Theorem one can see that 10 holds. Since $p<r$, applying the second case of Theorem 6, we obtain that

$$
\begin{aligned}
\mathcal{R}(p, r ; \tilde{v}, w) \approx( & \left.\left(\int_{0}^{\infty} v(s)^{p}\left(\int_{s}^{\infty} g\right) d s\right)^{\frac{r}{r-p}}\left(\int_{0}^{t} w^{r}\right)^{-\frac{r}{r-p}} w(t)^{r} d t\right)^{\frac{r-p}{r p}} \\
+ & \left(\int_{0}^{\infty} v(s)^{p}\left(\int_{s}^{\infty} g\right) d s\right)^{\frac{1}{p}}\left(\int_{0}^{\infty} w^{r}\right)^{-\frac{1}{r}} .
\end{aligned}
$$


Then, $C \approx C_{1}+C_{2}$, where

$$
C_{1}:=\sup _{g \in \mathfrak{M}^{+}} \frac{\left(\int_{0}^{\infty}\left(\int_{0}^{t} v(s)^{p}\left(\int_{s}^{\infty} g\right) d s\right)^{\frac{r}{r-p}}\left(\int_{0}^{t} w^{r}\right)^{-\frac{r}{r-p}} w(t)^{r} d t\right)^{\frac{r-p}{r p}}}{\|g\|^{\frac{1}{p}}}
$$

and

$$
C_{2}:=\left(\int_{0}^{\infty} w^{r}\right)^{-\frac{1}{r}} \sup _{g \in \mathfrak{M}^{+}} \frac{\left(\int_{0}^{\infty} v(s)^{p} \int_{s}^{\infty} g(y) d y d s\right)^{\frac{1}{p}}}{\|g\|^{\frac{1}{p}}} .
$$

First observe that, using Fubini's Theorem and duality principle one more time, we have

$$
C_{2}=\left(\int_{0}^{\infty} w^{r}\right)^{-\frac{1}{r}}\left(\int_{0}^{\infty}\left(\int_{0}^{y} v(s)^{p} d s\right)^{\frac{q}{p}} u(y)^{q} d y\right)^{\frac{1}{q}}
$$

and $C_{1}^{p}$ is the best constant in the inequality 9 with parameters $p=\frac{q}{q-p}$ and $q=\frac{r}{r-p}$, and weights

$$
\mathfrak{u}(s)=u(s)^{-p}, \quad \mathfrak{v}(s)=v(s)^{p}, \quad \mathfrak{w}(s)=\left(\int_{0}^{s} w^{r}\right)^{-1} w(s)^{r-p}, \quad s>0 .
$$

It remains to apply Theorem 7. To this end we should again split this case into two parts.

(i) If $r \leq q$, then applying the first case in Theorem 7 , we obtain that $C_{1} \approx I_{8}+I_{9}$ and the result follows.

(ii) If $q<r$, then applying the second case in Theorem 7 we obtain that $C_{1} \approx$ $I_{10}+I_{11}$ and the result follows.

Declaration of Competing Interests The author declares no competing interests.

Acknowledgement The author thanks the anonymous referees for their helpful remarks, which have improved the final version of this paper.

\section{REFERENCES}

[1] Astashkin, S. V., Maligranda, L., Structure of Cesàro function spaces, Indag. Math. (N.S.), 20(3) (2009), 329-379. https://dx.doi.org/10.1016/S0019-3577(10)00002-9

[2] Astashkin, S. V., Maligranda, L., Structure of Cesàro function spaces: a survey, Banach Center Publications, 102 (2014), 13-40. https://dx.doi.org/10.4064/bc102-0-1

[3] Barza, S., Marcoci, A. N., Marcoci, L. G., Factorizations of weighted Hardy inequalities, Bull. Braz. Math. Soc. (N.S.), 49(4) (2018), 915-932. https://dx.doi.org/10.1007/s00574-018-00877

[4] Bennett, G., Factorizing the classical inequalities, Mem. Amer. Math. Soc., 120 (576) (1996). https://dx.doi.org/10.1090/memo/0576

[5] Boas, R. P., Jr., Some integral inequalities related to Hardy's inequality, J. Analyse Math., 23 (1970), 53-63. https://dx.doi.org/10.1007/BF02795488 
[6] Carro, M., Gogatishvili, A., Martín, J., Pick, L., Weighted inequalities involving two Hardy operators with applications to embeddings of function spaces, J. Operator Theory, 59(2) (2008), 309-332.

[7] Evans, W. D., Gogatishvili, A., Opic, B., The reverse Hardy inequality with measures, Math. Inequal. Appl., 11(1) (2008), 43-74. https://dx.doi.org/10.7153/mia-11-03

[8] Gogatishvili, A., Mustafayev, R. Ch., Persson, L. -E., Some new iterated Hardy-type inequalities, J. Funct. Spaces Appl., Art. ID 734194, (2012). https://dx.doi.org/10.1155/2012/734194

[9] Gogatishvili, A., Mustafayev, R. Ch., Ünver, T., Embeddings between weighted Copson and Cesàro function spaces, Czechoslovak Math. J., 67(4) (2017), 1105-1132. https://dx.doi.org/10.21136/CMJ.2017.0424-16

[10] Gogatishvili, A., Mustafayev, R. Ch., Ünver, T., Pointwise multipliers between weighted Copson and Cesàro function spaces, Math. Slovaca, 69(6) (2019), 1303-1328. https://dx.doi.org/10.1515/ms-2017-0310

[11] Grosse-Erdmann, K.-G., The blocking technique, weighted mean operators and Hardy's inequality, Lecture Notes in Mathematics, Springer-Verlag, Berlin, 1998. https://dx.doi.org/10.1007/BFb0093486

[12] Hassard, B. D., Hussein, D. A., On Cesàro function spaces, Tamkang J. Math., 4 (1973), $19-25$.

[13] Kamińska, A., Kubiak, D., On the dual of Cesàro function space, Nonlinear Anal., 75(5) (2012), 2760-2773. https://dx.doi.org/10.1016/j.na.2011.11.019

[14] Kolwicz, P., Leśnik, K., Maligranda, L., Symmetrization, factorization and arithmetic of quasi-Banach function spaces, J. Math. Anal. Appl., 470(2) (2019), 1136-1166. https://dx.doi.org/10.1016/j.jmaa.2018.10.054

[15] Kufner, A., Persson, L. -E., Samko, N., Weighted Inequalities of Hardy Type, Second Ed., World Scientific Publishing Co. Pte. Ltd., Hackensack, NJ, 2017. https://dx.doi.org/10.1142/10052

[16] Leśnik, K., Maligranda, L., Abstract Cesàro spaces. Duality, J. Math. Anal. Appl., 424(2) (2015), 932-951. https://dx.doi.org/10.1016/j.jmaa.2014.11.023

[17] Leśnik, K., Maligranda, L., Abstract Cesàro spaces. Optimal range, Integral Equations Operator Theory, 81(2) (2015), 227-235. https://dx.doi.org/10.1007/s00020-014-2203-4

[18] Leśnik, K., Maligranda, L., Interpolation of abstract Cesàro, Copson and Tandori spaces, Indag. Math. (N.S.), 27(3) (2016), 764-785. https://dx.doi.org/10.1016/j.indag.2016.01.009

[19] Mustafayev, R. Ch., Ünver, T., Reverse Hardy-type inequalities for supremal operators with measures, Math. Inequal. Appl., 18(4) (2015), 1295-1311. https://dx.doi.org/10.7153/mia18-101

[20] Shiue, J. -S., A note on Cesàro function space, Tamkang J. Math., 1 (1970), 91-95.

[21] Sy, P. W., Zhang, W. Y., Lee, P. Y., The dual of Cesàro function spaces, Glas. Mat. Ser. III, $22(42)$ (1) (1987), 103-112.

[22] Tandori, K., Über einen speziellen Banachschen Raum, Publ. Math. Debrecen, 3 (1954), 263268. 